\title{
3 Research Square \\ Effects of first- and third-person perspectives created using a head-mounted display on dart- throwing accuracy
}

\author{
Yuki Ueyama ( $\sim$ ueyama@nda.ac.jp) \\ National Defense Academy of Japan https://orcid.org/0000-0002-1415-8875 \\ Masanori Harada \\ National Defense Academy of Japan
}

\section{Research Article}

Keywords: motor control, skill transfer, human performance, visual perspective, body ownership, agency

Posted Date: May 27th, 2021

DOl: https://doi.org/10.21203/rs.3.rs-564416/v1

License: (c) (1) This work is licensed under a Creative Commons Attribution 4.0 International License.

Read Full License

Version of Record: A version of this preprint was published at Virtual Reality on July 29th, 2021. See the published version at https://doi.org/10.1007/s10055-021-00562-x. 


\section{Abstract}

The first-person perspective (1PP) and third-person perspective (3PP) have both been adopted in video games. The 1PP can induce a strong sense of immersion, and the 3PP allows players to perceive distances easily. Virtual reality (VR) technologies have also adopted both perspectives to facilitate skill acquisition. However, how 1PP and 3PP views affect motor skills in the real world, as opposed to in games and virtual environments, remains unclear. This study examined the effects of the 1PP and 3PP on real-world dart-throwing accuracy after head-mounted display (HMD)-based practice tasks involving either the 1PP or 3PP. The 1PP group showed poorer dart-throwing performance, whereas the 3PP task had no effect on performance. Furthermore, while the effect of the 1PP task persisted for some time, that of task 3PP disappeared immediately. Therefore, the effects of 1PP VR practice tasks on motor control transfer more readily to the real world than do those of 3PP tasks.

\section{Introduction}

Video games use either a first-person perspective (1PP) or third-person perspective (3PP). The 1PP promotes immersion and allows performance of precision tasks, such as shooting. Meanwhile, the 3PP provides a wide field of view and allows easy interaction with the surrounding environment. Thus, although less-suited for precision tasks, the 3PP allows players to easily follow the movements of an avatar.

Virtual reality (VR) technologies have become popular for training materials in the fields of aviation (Hays et al. 1992), surgery (Hong et al. 2020; Seymour et al. 2002), and critical care (Koutitas et al. 2020), i.e., fields in which it is difficult to practice the necessary skills safely in the real world. Recently, VR technologies have also been applied to sports and skills training, and their ability to improve real-world performance has been demonstrated to some extent (Michalski et al. 2019; Osti et al. 2020). One study reported a marked improvement in motor skills in the real world following VR training (Gray 2017). According to previous studies, it is important that the virtual environment (VE) be as close as possible to the real-world setting to maximize the transfer of learning, where humans establish motor plans to optimize the sensory information presented during skills training. However, if the practice conditions change, a previously developed motor plan may no longer be appropriate (Miles et al. 2012). In addition, training in the VE is difficult for skills such as throwing that require precise motor coordination and control of force.

An investigation of the effects of the 1PP and 3PP on performance in the VE suggested that the 1PP promoted more accurate interactions with virtual objects, while the 3PP was associated with better spatial awareness (Gorisse et al. 2017). Research has focused on throwing tasks to compare the effects of different perspectives. One study compared the effects of head-mounted display (HMD)-based training using the 1PP and 3PP for a ball-catching task. That study reported that the subjects' performance after training under the 3PP condition was similar to their real-world performance after standard training. In contrast, performance after the 1PP training differed in certain respects from that after the 3PP and 
normal training, although there was no absolute performance difference among the training types (Salamin et al. 2010). Another study investigated how different types of visual feedback in a VE affected motor skills, as assessed by a basketball free-throw task (Covaci et al. 2015). Although the VE was associated with underestimation of distances, and the 1PP condition with disrupted distance perception, the performance under the 3PP condition was close to that in the real world. A recent study reported higher accuracy in throwing tasks with whole-body visualization in a VE (Pastel et al. 2020). Nevertheless, how 1PP and 3PP training affect motor performance in the real world, rather than in the VE, remains poorly understood.

The 3PP facilitates depth perception and real-time adjustment of the kinematics of throwing. Thus, although the 3PP is less-suited for precision tasks, we hypothesized that 3PP training could nevertheless facilitate precision throwing. To test this proposition, this study examined the effects of 1PP and 3PP HMD-based practice tasks on real-world dart-throwing ability. Precision throwing is a unique ability of humans (Roach et al. 2013) that has facilitated the development of cognitive abilities, which together with tool use provided an evolutionary advantage (Calvin 1982). Several factors have been shown to affect dart-throwing ability, including cognitive skills (Mendoza and Wichman 1978; Mosley et al. 2017), attentional focus (Lohse et al. 2010; Schorer et al. 2012), arm kinematics (Nasu et al. 2014; Tran et al. 2019), and release timing (Smeets et al. 2002). The mechanisms underlying motor skill acquisition have also been explored (Ikegami and Ganesh 2014; van Beers et al. 2013). A recent study demonstrated that adjustment of the kinematics of throwing may be the most important factor in the acquisition of new throwing skills, largely because this strategy is robust to temporal variability arising from neuromotor noise, whereas the strategy of adjusting release timing is more sensitive to that noise (Zhang et al. 2018). Neuromotor noise introduces uncertainty in movements (Ueyama 2014; Ueyama and Miyashita 2013), and robustness to this noise is important for the brain to optimally tune motor activity (Ueyama 2017a; Ueyama and Miyashita 2014).

In our experiment, subjects were asked to throw darts in a practice task using a 1PP or 3PP view, as provided by an HMD. Dart-throwing performance was compared before and after completing the 1PP or 3PP practice task. The results showed that dart-throwing performance did not change after the 3PP practice. However, the effect of the 1PP practice briefly persisted after removing the HMD, whereas that of the 3PP practice was immediately lost. Therefore, although our hypothesis was rejected, the results suggest that the degree of transfer of skills to the real world differs between the 1PP and 3PP views.

\section{Methods}

We divided the subjects into 1PP and 3PP groups. Each group threw a dart 60 times in total; the middle third of the throws were performed while wearing the HMD, which provided a 1PP or 3PP view (Fig. 1a-b; Electric Supplementary Material ESM_1.mp4). All 
experimental procedures were approved by the Ethics Review Board of the National Defense Academy of Japan.

\subsection{Subjects}

Forty academy students (two females) aged 19-23 years were recruited; six were lefthanded. All subjects had normal or corrected-to-normal vision, and reported no history of neurological or psychiatric, and provided written informed consent prior to inclusion in the study. They were randomly allocated to the 1 PP or 3PP group (both $n=20$ ).

\subsection{Apparatus}

A commercially available soft dartboard was used, positioned at a height of $1.73 \mathrm{~m}$. The distance from the throwing line to the dartboard was $2.44 \mathrm{~m}$ (Fig. 1c). The subjects threw standard plastic-tipped darts of the same size and weight. The HMD device was a Vive Pro (HTC, New Taipei, Taiwan; resolution: 1,400 × 1,600 pixels/eye; field of view: $110^{\circ}$; angular resolution: 14.5 pixels per degree). A stereo camera system $(640 \times 480$ pixels $)$ was mounted on the front of the HMD to provide the 1PP view (Fig 1d). For the 3PP view, a $360^{\circ}$ camera (Theta V; Ricoh, Tokyo, Japan) was set $1.70 \mathrm{~m}$ behind the throwing line on the right-hand side $\left(135^{\circ}\right)$. The resolution of the video streamed from the $360^{\circ}$ camera was $3,840 \times 1,920$ pixels, and the angular resolution was 5.3 pixels per degree. The body representation is often viewed from a distance in the 3PP, often from behind the body, or from the side or above (Seinfeld et al. 2020). We positioned the camera so that the arm posture and dartboard were not obscured from view by the subjects' body, similar to the camera position in some 3PP shooting video games (e.g., Biohazard 4-6; CAPCOM, Osaka, Japan). The HMD displayed images from the $360^{\circ}$ camera synchronized with the subjects' head movements, and the images were processed in real time using a 3D game engine (Unity; Unity Technologies, San Francisco, CA, USA). The view could be switched from the 1PP to the 3PP by the experimenter. The camera angles and heights were adjusted to match the centers of the dartboards in both the $1 \mathrm{PP}$ and $3 \mathrm{PP}$ views during throwing, and subjects were asked not to change their head position between the 1PP and 3PP views. The 
experiment was designed so that the subjects in both groups threw the darts in the same manner.

\subsection{Procedure}

The experiment was divided into three phases (pre-intervention, intervention, and postintervention). Each phase comprised five blocks of four dart throws (20 throws in total per phase). The subjects always threw the dart with their dominant hand and aimed for the center of the dartboard (i.e., bull's-eye). The distance from the bull's-eye to the dart, measured manually by the experimenter, served as the performance indicator. There was a 3-minute rest period between task phases; subjects could also rest while the measurements were being collected.

Before the experiment, the subjects threw 5-10 darts for familiarization with the laboratory environment (Fig. 1e). In the pre-intervention phase, the subjects were instructed to throw the dart at the bull's-eye 20 times, as accurately as possible. In the intervention phase, the subjects wore the HMD and threw 20 darts under the 1PP or 3PP condition. In the 3PP group, the 3PP view was only presented during throwing: the view was switched from the 1PP to the 3PP just prior to the dart-throw, when the subjects signaled that they were ready to throw, and returned to the 1PP view soon after the dart reached the dartboard. The effects of the intervention were measured during the postintervention phase. In this phase, the subjects removed the HMD and threw 20 darts in the same way as in the pre-intervention phase.

\subsection{Analysis}

We evaluated the effects of the 1PP and 3PP by comparing the dart-throwing performance between the pre- and post-intervention phases. We excluded the data of one 3PP group subject from the analysis because they failed to hit the dartboard twice during the preintervention phase. All other subjects hit the dartboard with all darts in the pre-intervention phase. Thus, the final analysis included the data of 20 and 19 subjects in the 1PP and 3PP groups, respectively. In the intervention and post-intervention phases, trials where the darts did not hit the dartboard or missed it by $>200 \mathrm{~mm}$, which is outside of the "play area" of 
the dartboard, were recorded as "failed trials." The failed trials were combined with 200mm misses in subsequent analyses.

\subsubsection{Model-based analysis}

We modeled the trial-by-trial performance of the 1PP and 3PP groups to elucidate the learning process in the VR practice tasks. Then, we assumed that the variability in dartthrowing performance converged in the pre-intervention phase (van Beers et al. 2013). Thus, differences in performance between the post- and pre-intervention phases were attributed to the intervention phase. The error on the $k^{\text {th }}$ trial, $y_{k^{\prime}}$ was defined as

$$
y_{k}=c \cdot e_{k}+d,
$$

where $c$ and $d$ are free parameters representing compliance and offset, respectively. The error $y_{k}$ is the absolute error, and indicates the distance from the bull's-eye to the dart. The error $e_{k}$ is the internal error for adaptation, and is denoted by

$$
e_{k}=\left|p_{k}-x_{k}\right|,
$$

where $p_{k}$ and $x_{k}$ are perturbation and memory, respectively. Subjects estimate perturbation $p_{k}$ through a motor adaptation process, similar to memory $x_{k}$, and the error $e_{k}$ affects their behavior directly. The perturbation $p_{k}$ is a binary signal induced by the $1 \mathrm{PP}$ or 3PP view, and took a value of 1 during the intervention phase and 0 at all other times:

$$
p_{k}=\left\{\begin{array}{ll}
1 & \text { intervention phase } \\
0 & \text { pre-/post-intervention phase }
\end{array} .\right.
$$

Here, $k=21,22, \ldots 40$ during the intervention phase. Any other values of $k$ indicate the preor post-intervention phase.

The memory, $x_{k}$, reflects the adaptation ratio for perturbation. It is assumed to be in an equilibrium state $x_{k}=0$ during the pre- and post-intervention phases, and is only 
affected by the error induced by perturbation (as a motor adaptation process) (Ueyama 2017b). We assumed that error was determined by memory and perceived as the prediction error $e_{k}$, and excluded execution noise because the brain seems to correct errors associated with previous motor planning rather than actual performance (van Beers 2009). We assumed that memory only decayed in the post-intervention phase after removing the perturbation, and had a different time constant from that of the intervention phase. Thus, memory updating is represented by

$$
x_{k+1}=\left\{\begin{array}{ll}
a_{1} \cdot x_{k}+b \cdot e_{k} & \text { intervention phase } \\
a_{2} \cdot x_{k} & \text { pre-/post-intervention phase }
\end{array}=\right.
$$

where $a_{1}$ and $a_{2}$ are the retention rates during the intervention phase and at all other times, respectively, and $b$ is the rate of adaptation to the perturbation in the intervention phase. The retention rate $a_{2}$ indicates the magnitude of after-effects of the intervention phase.

The five parameters $a_{1}, a_{2}, b, c$, and $d$ were estimated to fit the model to the experimental data, while minimizing the squared error using the interior-point method with the constraints $0 \leq a_{1}, a_{2}, b, c \leq 1$. The coefficient of determination $R^{2}$ was calculated in the standard way.

\subsubsection{Statistical analysis}

Means and standard deviations (SDs) were calculated for all 20 trials, for each subject, in the pre- and post-intervention phases, and the difference was evaluated using a two-tailed paired $t$-test (as a measure of the effects of the intervention phase). The data of the 1PP and 3PP groups were compared using a two-tailed Welch's $t$-test.

A standard bootstrap technique was applied to generate confidence intervals for trialby-trial performance. Then, we resampled the data 200 times, deriving mean values for each resampling.

\subsection{Validation of size and distance perception}


We were concerned about the validity of size and distance perception in our study because the $3 \mathrm{PP}$ view in our experiment was not stereoscopic and the 1PP stereo camera had a lower resolution than the $3 \mathrm{PP} 360^{\circ}$ camera. Therefore, we performed an additional experiment to validate the perception of distance and size in 1PP and 3PP.

We included 10 healthy subjects (age: $21-29$ years; height: $1.65-1.82 \mathrm{~m}$; all males) in this experiment who were not part of the main experiment. These subjects observed white paper cubes in either 1PP or 3PP view created by the HMD. They were asked to open their thumb and index fingers to imitate grasping of the cubes. We measured the width between their thumb and index finger manually, as a measure of the perceived size of the cubes. Each cube was displayed with one of the following side lengths: 50, 60, 70, 85, or $100 \mathrm{~mm}$ (Fig. 2a). Each cube was displayed on a cork sheet at a height of $0.95 \mathrm{~m}$ and distance of $0.80,1.60$, or $2.40 \mathrm{~m}$ (Fig. $2 \mathrm{~b}$ ). The subjects were required to reply with their estimated size of the cubes 30 times ( 2 perspectives $\times 3$ distances $\times 5$ sizes). Half of the subjects performed this task in $1 \mathrm{PP}$ view before the 3PP view, and the other half performed the task in the reverse order. The cubes were displayed at each location in a random manner. The HMD images were blacked out before and after each trial. After observing the cubes of all sizes at each location, we asked the subjects to estimate the number of footsteps (until the tenth place) required to reach the cube. The number of steps was used to calculate the perceived distance by a simple formula (distance $=$ steps $\times$ height $\times 0.37$ ) involving the step length, step height, and a slow walking speed.

We calculated differences in size and distance between the subjects' reports and the actual values. We performed a two-way repeated-measures ANOVA ( 2 perspectives $\times 3$ distances) to evaluate the effects of the 1PP and 3PP on these errors. Scheffe's multiple comparison test was used for the post hoc analysis, with an alpha level at 0.05.

\section{Results}

\subsection{Trial-by-trial performance}

The 1PP and 3PP groups showed constant errors during the pre-intervention phase (Fig. 3a). There was no significant difference in dart-throwing performance between the 1PP and 3PP groups during the preintervention phase ( $p=0.93$; Fig. $4 \mathrm{a})$. The error magnitude increased in both groups at the beginning of 
the intervention phase and then gradually decreased. The number of errors continued to decrease gradually in the 1PP group during the post-intervention phase, but not in the 3PP group. Memory decayed more slowly during the post-intervention phase in the 1PP than 3PP group (Fig. 3b), i.e., the postintervention phase $a_{2}$ values were $0.83\left(R^{2}=0.72\right)$ and $0.22\left(R^{2}=0.91\right)$, respectively (Table 1$)$. The mean retention rate was significantly higher in the 1PP group than the 3PP group $(0.55 \pm 0.35$ and $0.31 \pm 0.32$ $\mathrm{mm}$, respectively; $p=0.035$; Fig. $3 \mathrm{c}$ ).

\subsection{Comparison of the pre- and post-intervention phase dart-throwing performance}

We compared the error between the pre- and post-intervention phases to evaluate the effects of the intervention (Fig. 4a). In the 1PP group, the mean post-intervention phase error magnitude was larger than that during the pre-intervention phase $(66.9 \pm 10.3$ and $60.2 \pm 7.1 \mathrm{~mm}$, respectively; $p=0.0046)$. In contrast, the 3PP group showed a slightly, but not significantly, smaller mean error magnitude postversus pre-intervention ( $57.8 \pm 8.1$ and $60.4 \pm 7.0 \mathrm{~mm}$, respectively; $p=0.21)$.

The 1PP and 3PP HMD practice tasks had different effects on performance in the post-intervention phase. The mean changes in performance, computed by subtracting pre-intervention phase scores from those in the post-intervention phase, were $6.7 \pm 9.6$ and $-2.6 \pm 8.9 \mathrm{~mm}$ in the 1PP and 3PP groups, respectively; this difference was significant ( $p=0.0026$; Fig. $4 b)$.

\subsection{Size and distance perception}

In the HMD-based tasks, there was a difference in mean size perception values between 1PP and 3PP only for near objects (Fig. 5a; 1PP: $-0.8 \pm 9.0,5.5 \pm 6.5$, and $2.9 \pm 6.5$; 3PP: $-10.5 \pm 5.9,4.7 \pm 8.6$, and $1.2 \pm$ $6.1 \mathrm{~mm}$ for near [0.8 m], moderate [1.6 m], and far [2.4 m] distances, respectively). The two-way repeated ANOVA revealed significant main effects of perspective $\left(F_{(1,9)}=8.32, p=0.018, \eta^{2}=0.46\right)$ and distance $\left(F_{(1,9)}=6.38, p=0.032, \eta^{2}=0.27\right)$, and a significant interaction effect $\left(F_{(1,9)}=5.83, p=0.039, \eta^{2}=0.26\right)$. Using Scheffe's test, we found a significant difference between the near distance and other distances (moderate: $p=0.050$; far: $p=0.0016$ ) in 3PP. The difference between the distances was not statistically significant in 1PP $(p>0.20)$. There was a difference between both perspectives for the near distance $(p=$ $0.0079)$, but not for the other distances $(p>0.45)$.

The mean error and variance of the perceived distance gradually increased with an increase in the actual distance in 1PP and 3PP (1PP: $0.14 \pm 0.26,0.18 \pm 0.60$, and $0.72 \pm 0.85$; 3PP: $-0.15 \pm 0.28,0.20 \pm 0.43$, and $0.81 \pm 0.67 \mathrm{~m}$ for near, moderate, and far distances, respectively; Fig. $5 \mathrm{~b}$ ). Two-way repeated ANOVA revealed a significant main effect of distance $\left(F_{(1,9)}=6.56, p=0.031, \eta^{2}=0.42\right)$ and a significant interaction effect $\left(F_{(1,9)}=7.62, p=0.022, \eta^{2}=0.39\right)$, but there was no significant main effect of perspective $\left(F_{(1,9)}=4.25, p=0.069, \eta^{2}=0.19\right)$. Scheffe's test showed significant differences between the 
pairs of distances in 1PP and 3PP ( $p<0.05)$, except for near and moderate distances in 1PP $(p=0.17)$. There were no significant differences between 1PP and 3PP for any distance $(p>0.65)$.

\section{Discussion}

The objective of this study was to clarify the effects of practice HMD-based tasks with a 1PP and 3PP view on a real-world skill, i.e., dart throwing. Subjects' performance declined while wearing the HMD regardless of the type of perspective, but showed gradual adaptions to the environment. The effects of the practice task persisted after removal of the HMD only in the 1PP group, who showed poorer performance than before the intervention. In contrast, the 3PP group showed almost the same performance before and after the HMD intervention. This effect was not caused by simple visual displacement, because it was reported that a large amount of visual displacement induced a greater decrease in adaptation magnitude but showed a larger after effect (Lee and Park 2020). Thus, while the 1PP HMD practice task affected real-world performance, the 3PP task did not. According to the modelbased analysis, the effect of the 1PP HMD practice persisted for a while, reflected in the higher retention rate of that group compared to the 3PP group; in the latter group, the effect of the practice disappeared immediately. The difference in perception of size and distance between 1PP and 3PP was minimal, except for distances less than $1.6 \mathrm{~m}$. Because the dartboard was set $2.44 \mathrm{~m}$ from the subjects, according to the convention for soft darts games, we believe that differences in the perception of size and distance between 1PP and 3PP had little effect on our results.

The 1PP might be preferable for inducing a sense of ownership of a virtual body (i.e., avatar) in a VE (Slater et al. 2010), although both the IPP and 3PP can induce a strong feeling of spatial presence (Gorisse et al. 2017). It appears that, via the 1PP, a sense of body ownership can be transferred to others' bodies (i.e., VR body swapping) (Petkova and Ehrsson 2008). In contrast, with the 3PP, a sense of body ownership may be absent, such that sensations are experienced as if they are outside the body (Ehrsson 2007). Even when different techniques are used to induce a sense of body ownership in 3PP, the sense of body ownership remains stronger in 1PP (Galvan Debarba et al. 2017). The sense of body ownership interacts with the sense of agency, by which some actions are self-generated (Arzy and Schacter 2019). The sense of agency arises directly from an internal model (Engbert et al. 2008; Moore and Haggard 2008; Moore et al. 2009; Vogeley and Fink 2003), represented in the cerebellum and associated with motor adaptation (Wolpert et al. 1998). Thus, the difference in the effects of the 1PP and 3PP practice tasks in this study might be attributable to the presence (or absence) of senses of agency and body ownership.

The performance of the 1PP group decreased during the intervention phase, even though the visual information provided therein was the same as in the pre-intervention phase. A previous study reported a similar result (Schorer et al. 2012). Although visual displacement problems are caused by the distance between a front-mounted camera and the human eye inducing visuomotor performance deterioration (Lee and Park 2020), our apparatus is designed to suppress this problem by matching the positions of the camera and the eyes. Therefore, this finding could be explained by two factors. First, some subjects 
had to change the kinematics of their throwing style when wearing the HMD; some held their hands in front of their face in the pre-intervention phase, but could not assume the same posture in the intervention phase because of the HMD. Second, because the resolution of the camera was very low compared to that of the HMD, the 1PP group was essentially rendered near-sighted. A previous study reported that 3PP practice had a similar effect on real-world performance to the 1PP practice (Salamin et al. 2010). Our results seem to contradict this, where the effects of the 3PP practice disappeared immediately while and those of the 1PP practice persisted. We suggest that the reason why the 3PP and 1PP practices had similar effects on performance in the previous study was that, while the effects of the 3PP practice disappeared immediately, any advantage of the 1PP practice would have been negated by issues with the HMDs, as in our experiment.

Although a $360^{\circ}$ camera was used to create the 3PP view in this study, the camera could not reproduce stereoscopic vision. The perception of size and distance (except near distance) is likely similar between the 1PP and 3PP views. Therefore, stereoscopic vision likely would have reduced error during the intervention phase, but we suggest that practice does not affect real-world performance, as indicated by this result, due to the lack of a sense of body ownership.

In summary, the 3PP and 1PP HMD-based practice tasks had different effects on dart-throwing performance. Although the effects of the 1PP task persisted for some time after removing the HMD, those of the 3PP task did not. Therefore, we suggest that 1PP practice tasks can affect real-world motor control, while the effects of 3PP practice tasks may be limited by the lack of any sense of agency and body ownership.

\section{Declarations}

\section{Author contributions}

Y.U. designed the study, performed the experiments, collected and analyzed the data, and wrote the manuscript. Y.U. and M.H. discussed the results, and approved the final manuscript.

Acknowledgements

We would like to thank Yu Maeda for his help in the experiments. A part of this work was supported by Hayao Nakayama Foundation for Science \& Technology and Culture under grant number R1-A1-32, and JSPS KAKENHI under grant numbers JP16K12518 and JP19K20745.

\section{References}

Arzy S, Schacter DL (2019) Self-Agency and Self-Ownership in Cognitive Mapping Trends in Cognitive Sciences 23:476-487 doi:https://doi.org/10.1016/j.tics.2019.04.003 
Calvin WH (1982) Did throwing stones shape hominid brain evolution? Ethology and Sociobiology 3:115124 doi:https://doi.org/10.1016/0162-3095(82)90010-3

Covaci A, Olivier A, Multon F (2015) Visual Perspective and Feedback Guidance for VR Free-Throw Training leee Comput Graph 35:55-65 doi:10.1109/MCG.2015.95

Ehrsson HH (2007) The Experimental Induction of Out-of-Body Experiences Science 317:1048-1048 doi:10.1126/science. 1142175

Engbert K, Wohlschläger A, Haggard P (2008) Who is causing what? The sense of agency is relational and efferent-triggered Cognition 107:693-704 doi:https://doi.org/10.1016/j.cognition.2007.07.021

Galvan Debarba H, Bovet S, Salomon R, Blanke O, Herbelin B, Boulic R (2017) Characterizing first and third person viewpoints and their alternation for embodied interaction in virtual reality PLOS ONE 12:e0190109 doi:10.1371/journal.pone.0190109

Gorisse G, Christmann O, Amato EA, Richir S (2017) First- and Third-Person Perspectives in Immersive Virtual Environments: Presence and Performance Analysis of Embodied Users Frontiers in Robotics and Al 4 doi: $10.3389 /$ frobt. 2017.00033

Gray R (2017) Transfer of Training from Virtual to Real Baseball Batting Frontiers in Psychology 8 doi:10.3389/fpsyg.2017.02183

Hays RT, Jacobs JW, Prince C, Salas E (1992) Flight Simulator Training Effectiveness: A Meta-Analysis Military Psychology 4:63-74 doi:10.1207/s15327876mp0402_1

Hong M, Rozenblit JW, Hamilton AJ (2020) Simulation-based surgical training systems in laparoscopic surgery: a current review Virtual Reality doi:10.1007/s10055-020-00469-z

Ikegami T, Ganesh G (2014) Watching novice action degrades expert motor performance: causation between action production and outcome prediction of observed actions by humans Sci Rep 4:6989 doi:10.1038/srep06989

Koutitas G, Smith S, Lawrence G (2020) Performance evaluation of AR/VR training technologies for EMS first responders Virtual Reality doi:10.1007/s10055-020-00436-8

Lee JH, Park J-H (2020) Visuomotor adaptation to excessive visual displacement in video see-through HMDs Virtual Reality 24:211-221 doi:10.1007/s10055-019-00390-0

Lohse KR, Sherwood DE, Healy AF (2010) How changing the focus of attention affects performance, kinematics, and electromyography in dart throwing Hum Movement Sci 29:542-555 doi:https://doi.org/10.1016/j.humov.2010.05.001 
Mendoza D, Wichman H (1978) "Inner" Darts: Effects of Mental Practice on Performance of Dart Throwing Percept Mot Skills 47:1195-1199 doi:10.2466/pms.1978.47.3f.1195

Michalski SC, Szpak A, Loetscher T (2019) Using Virtual Environments to Improve Real-World Motor Skills in Sports: A Systematic Review Frontiers in Psychology 10 doi:10.3389/fpsyg.2019.02159

Miles HC, Pop SR, Watt SJ, Lawrence GP, John NW (2012) A review of virtual environments for training in ball sports Comput Graph 36:714-726

Moore J, Haggard P (2008) Awareness of action: Inference and prediction Conscious Cogn 17:136-144 doi:https://doi.org/10.1016/j.concog.2006.12.004

Moore JW, Wegner DM, Haggard P (2009) Modulating the sense of agency with external cues Conscious Cogn 18:1056-1064 doi:https://doi.org/10.1016/j.concog.2009.05.004

Mosley E, Laborde S, Kavanagh E (2017) The contribution of coping related variables and cardiac vagal activity on the performance of a dart throwing task under pressure Physiol Behav 179:116-125 doi:10.1016/j.physbeh.2017.05.030

Nasu D, Matsuo T, Kadota K (2014) Two Types of Motor Strategy for Accurate Dart Throwing PLOS ONE 9:e88536 doi:10.1371/journal.pone.0088536

Osti F, de Amicis R, Sanchez CA, Tilt AB, Prather E, Liverani A (2020) A VR training system for learning and skills development for construction workers Virtual Reality doi:10.1007/s10055-020-00470-6

Pastel S, Chen C-H, Petri K, Witte K (2020) Effects of body visualization on performance in head-mounted display virtual reality PLOS ONE 15:e0239226 doi:10.1371/journal.pone.0239226

Petkova VI, Ehrsson HH (2008) If I Were You: Perceptual Illusion of Body Swapping PLOS ONE 3:e3832 doi:10.1371/journal.pone.0003832

Roach NT, Venkadesan M, Rainbow MJ, Lieberman DE (2013) Elastic energy storage in the shoulder and the evolution of high-speed throwing in Homo Nature 498:483-486 doi:10.1038/nature12267

Salamin P, Tadi T, Blanke O, Vexo F, Thalmann D (2010) Quantifying Effects of Exposure to the Third and First-Person Perspectives in Virtual-Reality-Based Training IEEE Transactions on Learning Technologies 3:272-276 doi:10.1109/TLT.2010.13

Schorer J, Jaitner T, Wollny R, Fath F, Baker J (2012) Influence of varying focus of attention conditions on dart throwing performance in experts and novices Exp Brain Res 217:287-297 doi:10.1007/s00221-0112992-5

Seinfeld S, Feuchtner T, Maselli A, Müller J (2020) User Representations in Human-Computer Interaction Human-Computer Interaction:1-39 doi:10.1080/07370024.2020.1724790 
Seymour NE, Gallagher AG, Roman SA, O’Brien MK, Bansal VK, Andersen DK, Satava RM (2002) Virtual Reality Training Improves Operating Room Performance: Results of a Randomized, Double-Blinded Study Ann Surg 236:458-464

Slater M, Spanlang B, Sanchez-Vives MV, Blanke O (2010) First Person Experience of Body Transfer in Virtual Reality PLOS ONE 5:e10564 doi:10.1371/journal.pone.0010564

Smeets JBJ, Frens MA, Brenner E (2002) Throwing darts: timing is not the limiting factor Exp Brain Res 144:268-274 doi:10.1007/s00221-002-1072-2

Tran BN, Yano S, Kondo T (2019) Coordination of human movements resulting in motor strategies exploited by skilled players during a throwing task PLOS ONE 14:e0223837 doi:10.1371/journal.pone.0223837

Ueyama Y (2014) Mini-max feedback control as a computational theory of sensorimotor control in the presence of structural uncertainty Front Comput Neurosci 8:119 doi:10.3389/fncom.2014.00119

Ueyama Y (2017a) Optimal feedback control to describe multiple representations of primary motor cortex neurons J Comput Neurosci 43:93-106 doi:10.1007/s10827-017-0650-z

Ueyama Y (2017b) System identification of neural mechanisms from trial-by-trial motor behaviour: modelling of learning, impairment and recovery Adv Robotics 31:107-117 doi:10.1080/01691864.2016.1266966

Ueyama Y, Miyashita E (2013) Signal-Dependent Noise Induces Muscle Co-Contraction to Achieve Required Movement Accuracy: A Simulation Study with an Optimal Control Curr Bioinform 8:16-24 doi:10.2174/1574893611308010005

Ueyama Y, Miyashita E (2014) Optimal Feedback Control for Predicting Dynamic Stiffness During Arm Movement IEEE Trans Ind Electron 61:1044-1052 doi:10.1109/Tie.2013.2273473

van Beers RJ (2009) Motor Learning Is Optimally Tuned to the Properties of Motor Noise Neuron 63:406417 doi:https://doi.org/10.1016/j.neuron.2009.06.025

van Beers RJ, van der Meer Y, Veerman RM (2013) What Autocorrelation Tells Us about Motor Variability: Insights from Dart Throwing PLOS ONE 8:e64332 doi:10.1371/journal.pone.0064332

Vogeley K, Fink GR (2003) Neural correlates of the first-person-perspective Trends in Cognitive Sciences 7:38-42 doi:https://doi.org/10.1016/S1364-6613(02)00003-7

Wolpert DM, Miall RC, Kawato M (1998) Internal models in the cerebellum Trends in Cognitive Sciences 2:338-347 doi:https://doi.org/10.1016/S1364-6613(98)01221-2 
Zhang Z, Guo D, Huber ME, Park S-W, Sternad D (2018) Exploiting the geometry of the solution space to reduce sensitivity to neuromotor noise PLoS Comput Biol 14:e1006013 doi:10.1371/journal.pcbi.1006013

\section{Tables}

Table 1. Estimated values of the model parameters fitted to the experimental data, as indicators of trial-by-trial performance.

\begin{tabular}{llccccc}
\hline & $a_{1}$ & $a_{2}$ & $b$ & $c$ & $d$ \\
\hline $1 \mathrm{PP}$ & Mean behavior & 0.95 & 0.83 & 0.071 & 0.040 & 0.060 \\
& Subject mean \pm SD & $0.67 \pm 0.37$ & $0.55 \pm 0.35$ & $0.41 \pm 0.34$ & $0.058 \pm 0.032$ & $0.060 \pm 0.012$ \\
\hline 3PP & Mean behavior & 0.81 & 0.22 & 0.14 & 0.090 & 0.058 \\
& Subject mean \pm SD & $0.61 \pm 0.34$ & $0.31 \pm 0.32$ & $0.32 \pm 0.28$ & $0.100 \pm 0.031$ & $0.056 \pm 0.014$ \\
\hline
\end{tabular}

\section{Figures}

a First-person perspective (1PP)

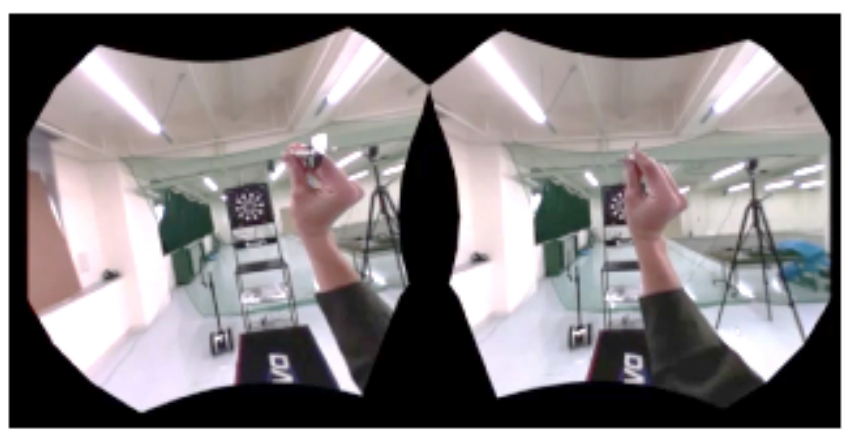

c

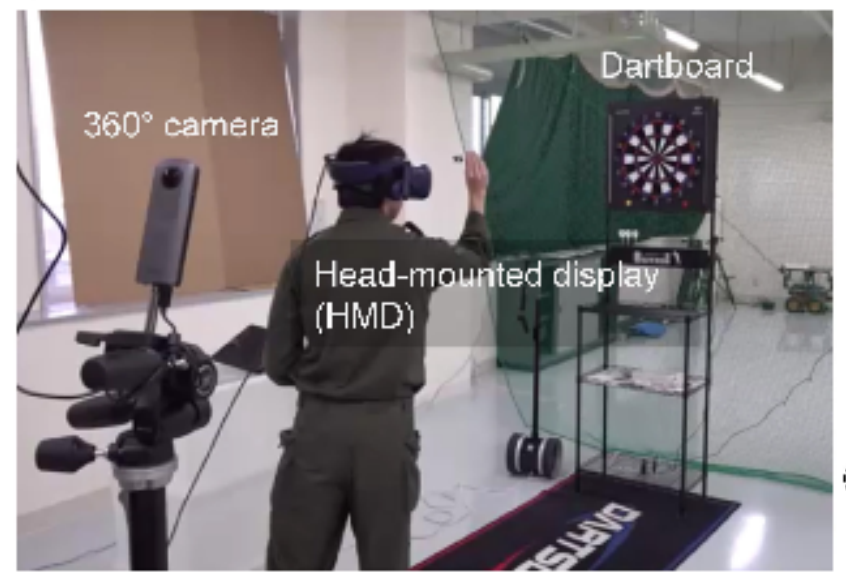

b Third-person perspective (3PP)

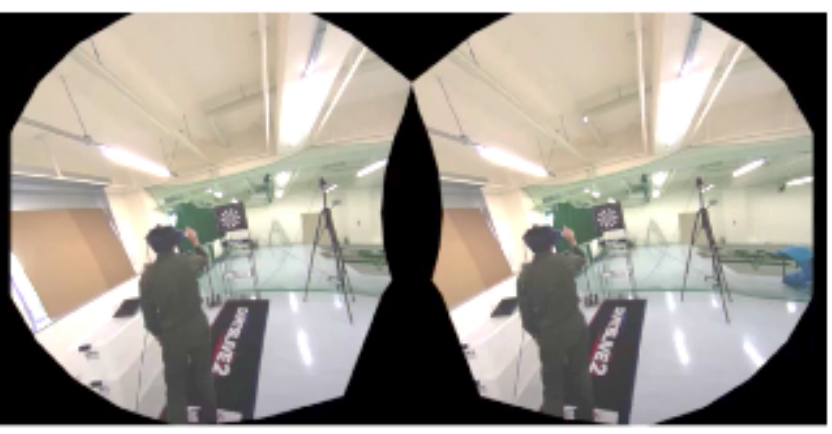

d

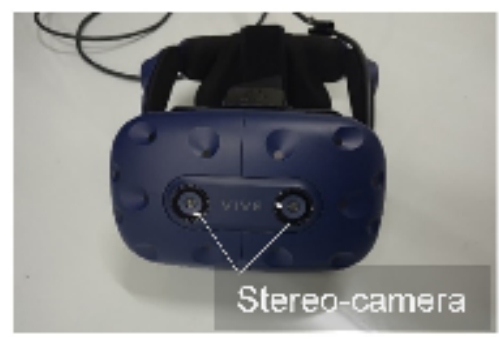

e

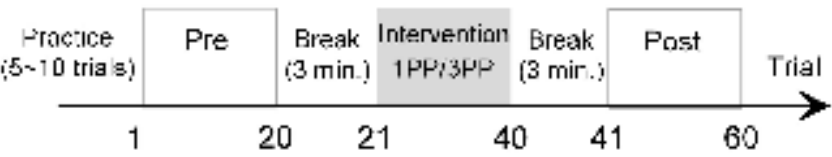

Page 15/18 
Figure 1

Experimental setup. (a) First-person perspective (1PP) and (b) third-person perspective (3PP) views. The left and right images in each panel are projected to the respective eye of the participant through a headmounted display (HMD). (c) Experimental apparatus and environment. (d) The stereo camera system mounted on the front of the HMD. (e) Experimental procedure.

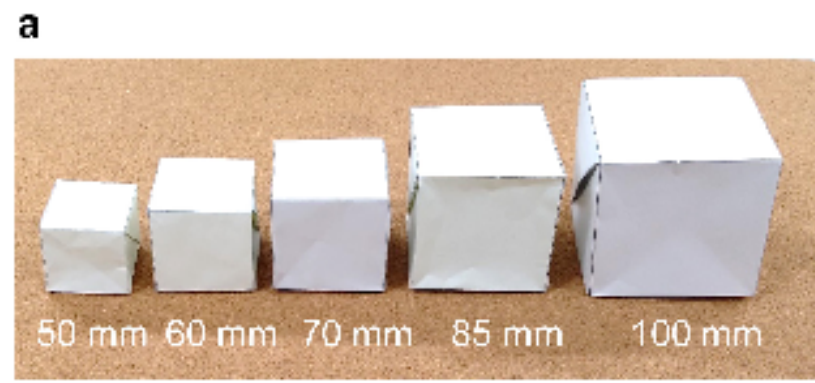

b

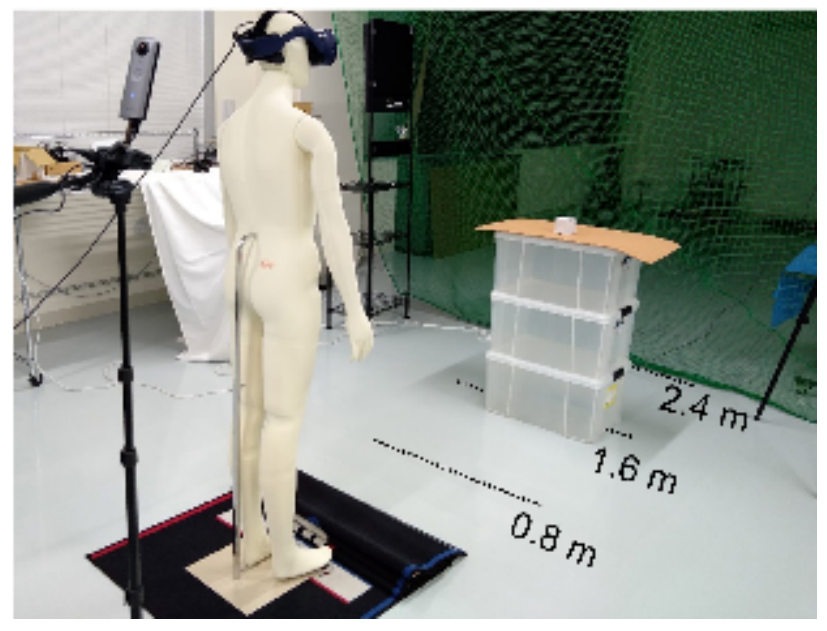

Figure 2

Validation experiment for size and distance perception. (a) Five cubes differing in size were presented to the subjects. (b) Experimental setup. The cubes were located at one of three distances (near: $0.8 \mathrm{~m}$; moderate: $1.6 \mathrm{~m}$; far: $2.4 \mathrm{~m}$ ) from the subjects. There were no marks or symbols on the floor to indicate the distance. 

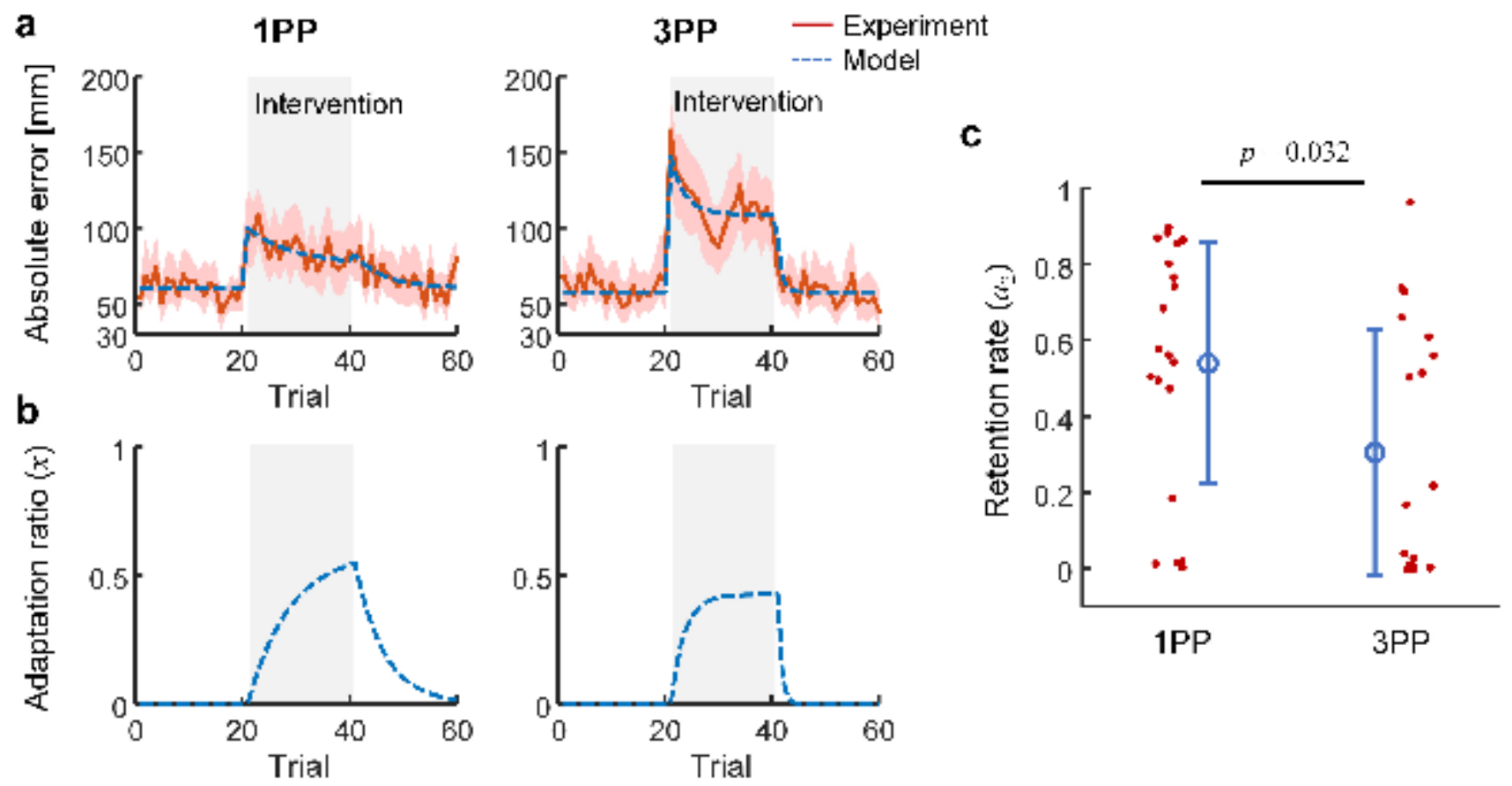

\section{Figure 3}

Dart-throwing performance according to model-based analysis. (a) Trial-by-trial performance across subjects. The red line and shaded area indicate mean and 95\% confidence interval data, respectively, derived from the experiments. The blue dotted line shows the model estimates. (b) Adaptation ratios. (c) Comparison of the mean retention rates of the 1PP and 3PP groups during the post-intervention phase. The vertical bars are standard deviations (SDs). The red dots show the data of individual subjects. The pvalue above the horizontal line is for the retention rate comparison between the 1PP and 3PP groups.
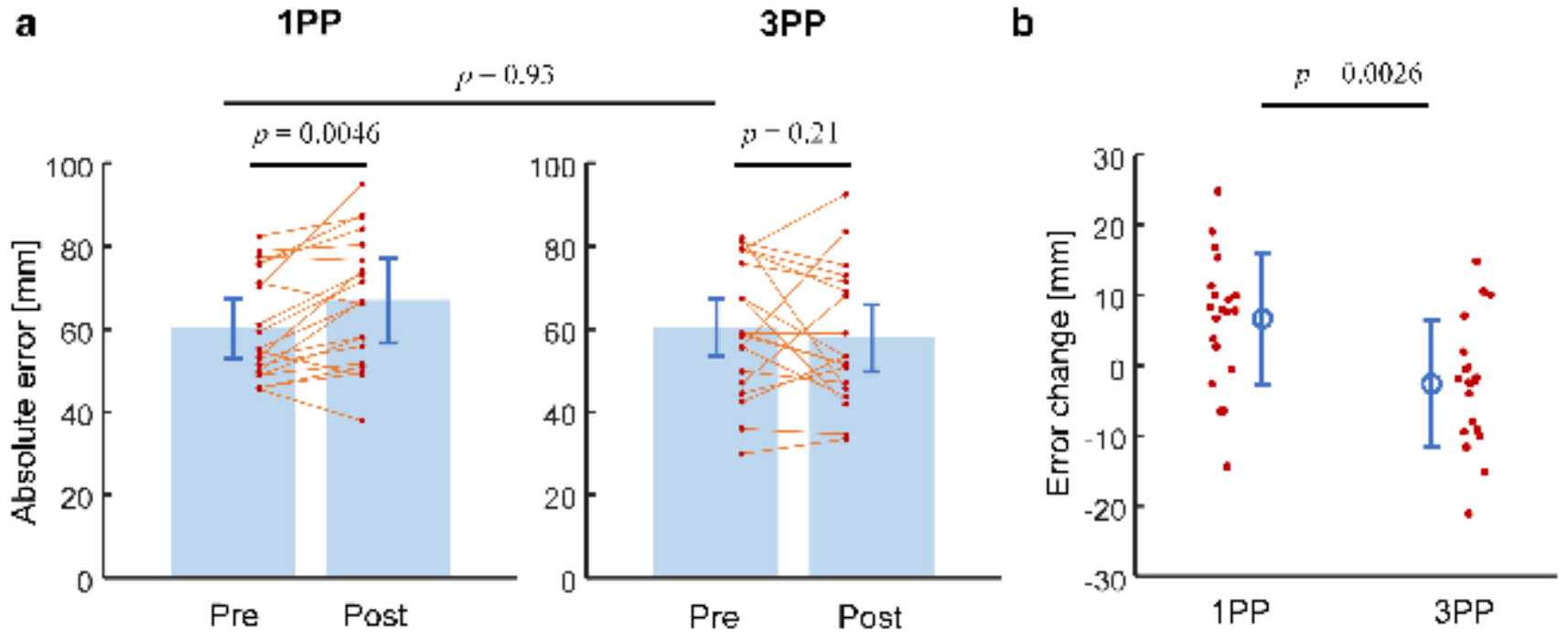

\section{Figure 4}

Comparison of performance between the pre- and post-intervention phases. The vertical bars indicate the SDs. The red dots show the data of individual subjects. The p-values above the horizontal lines are for 
the comparisons of pre- and post-intervention error, or change in error, between or within groups. (a) Mean absolute errors. (b) Changes in performance from the pre-intervention phase to the post-intervention phase.

a

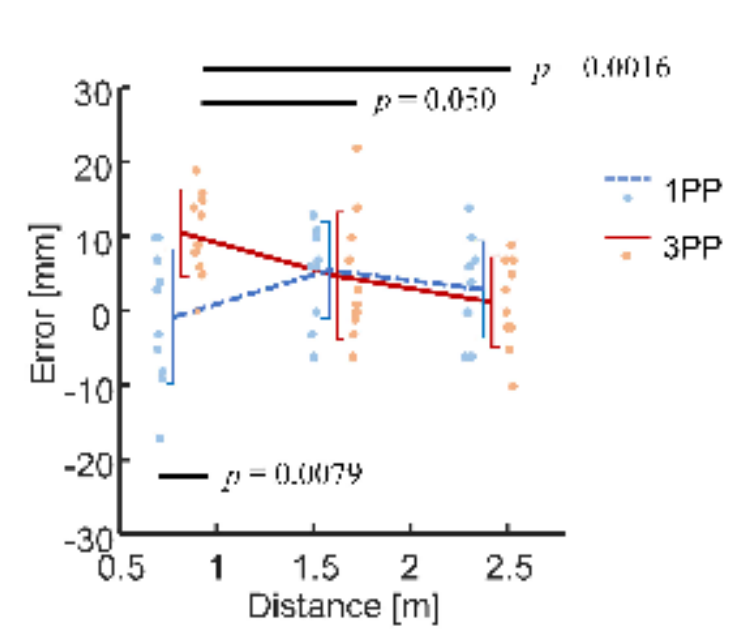

b Perceived distance

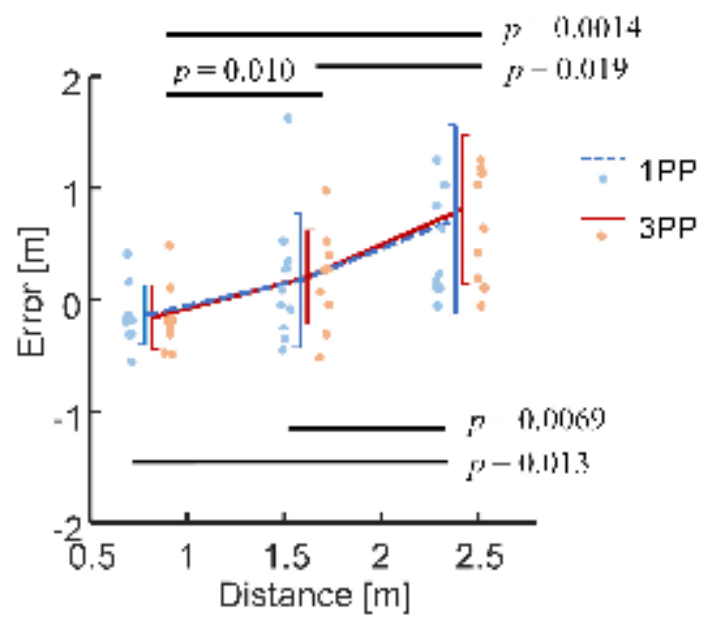

\section{Figure 5}

Effects of perspective on (a) size and (b) distance perception. The lines and vertical bars indicate the mean and SD, respectively. The dots show the data of individual subjects. The horizontal bars and accompanying values indicate the statistical differences and p-values computed by Scheffe's test.

\section{Supplementary Files}

This is a list of supplementary files associated with this preprint. Click to download.

- ESM1.mp4

- ESM2.xIsx 